\title{
THE EFFECTS OF PALM OIL TOCOTRIENOL-RICH FRACTION SUPPLEMENTATION ON BIOCHEMICAL PARAMETERS, OXIDATIVE STRESS AND THE VASCULAR WALL OF STREPTOZOTOCIN-INDUCED DIABETIC RATS
}

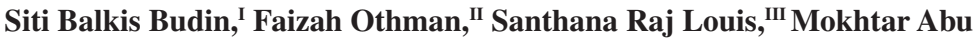 \\ Bakar, ${ }^{\text {IV }}$ Srijit Das, ${ }^{\text {II Jamaludin Mohamed }}{ }^{\mathrm{I}}$
}

doi: $10.1590 / \mathrm{S} 1807-59322009000300015$

\begin{abstract}
Budin SB, Othman F, Louis SR Bakar MA, Das S, Mohamed J. The effects of palm oil tocotrienol-rich fraction supplementation on biochemical parameters, oxidative stress and the vascular wall of streptozotocin-induced diabetic rats. Clinics. 2009;64(3):235-44.
\end{abstract}

OBJECTIVE: This study examined the effects of palm oil tocotrienol-rich fractions on streptozotocin-induced diabetic rats. METHODS: Animals were divided into three groups: (i) normal non-diabetic (NDM), (ii) diabetic treated (tocotrienol-rich fractions - TRF) and (iii) diabetic untreated (non-TRF). The treatment group received oral administration of tocotrienol-rich fractions (200 mg/kg body weight) daily for eight weeks. The normal non-diabetic and the diabetic untreated groups were fed standard rat feed. Blood glucose and lipid profiles, oxidative stress markers and morphological changes of the thoracic aorta were evaluated.

RESULTS: Tocotrienol-rich fractions treatment reduced serum glucose and glycated hemoglobin concentrations. The tocotrienolrich fractions group also showed significantly lower levels of plasma total cholesterol, low-density lipoprotein cholesterol, and triglyceride, as compared to the untreated group. The tocotrienol-rich fractions group had higher levels of high-density lipoprotein cholesterol, as compared to the untreated group. Superoxide dismutase activity and levels of vitamin C in plasma were increased in tocotrienol-rich fractions-treated rats. The levels of plasma and aorta malondealdehyde + 4-hydroxynonenal (MDA + 4-HNE) and oxidative DNA damage were significant following tocotrienol-rich fractions treatment. Electron microscopic examination showed that the normal morphology of the thoracic aorta was disrupted in STZ-diabetic rats. Tocotrienol-rich fractions supplementation resulted in a protective effect on the vessel wall.

CONCLUSION: These results show that tocotrienol-rich fractions lowers the blood glucose level and improves dyslipidemia. Levels of oxidative stress markers were also reduced by administration of tocotrienol-rich fractions. Vessel wall integrity was maintained due to the positive effects mediated by tocotrienol-rich fractions.

KEYWORDS: Tocotrienol-rich fraction; Antioxidant; Diabetes mellitus; Oxidative stress; Dyslipidemia; Vascular morphology.

\section{INTRODUCTION}

According to the World Health Organization (WHO), 170

\footnotetext{
'Department of Biomedical Science, Faculty of Allied Health Sciences, Universiti Kebangsaan - Malaysia.

IDepartment of Anatomy, Faculty of Medicine, Universiti Kebangsaan Malaysia - Malaysia,

IIDivision of Electron Microscope, Institute for Medical Research - Malaysia. ${ }^{\mathrm{IV}}$ Department of Pathology, Faculty of Medicine, Universiti Kebangsaan Malaysia - Malaysia.

Tel.: 60340405645

Email: amira@medic.ukm.my

Received for publication on September 29, 2008

Accepted for publication on November 24, 2008
}

million people were affected by diabetes in the year 2002, and this number is likely to increase to 366 million by the year 2030. ${ }^{1}$ Diabetes mellitus (DM) is a complex, progressive disease, which is accompanied by multiple complications. It has been recognized as the sole independent risk factor for the development of any cardiovascular disease. ${ }^{2}$ Cardiovascular complications include stroke and myocardial infarction, which are considered to be important causes of health-related deaths in diabetic patients. Alarmingly, literature statistics indicate that atherosclerosis accounts for about 8 to $10 \%$ of all diabetic deaths. ${ }^{3}$

Hyperglycemia has been accepted as an essential factor in 
the development of diabetic complications. ${ }^{4}$ Oxidative stress is significantly increased in DM, due to prolonged exposure to hyperglycemia. Oxidative stress is a common feature of DM, in which the activity of the antioxidant system is overwhelmed by excessive ROS production. ${ }^{5}$ Chronic hyperglycemia increases the generation of free radicals through non-enzymatic glycosylation, glucose autoxidation and increased activity in polyol pathways. ${ }^{6}$ Disturbances in capacities of the antioxidant defense system include scavenging enzymes such as superoxide dismutase (SOD) and glutathione reductase and deficiencies of antioxidants such as vitamin $\mathrm{C}$ and $\mathrm{E} .{ }^{7,8}$

The stability and capacity of antioxidants involved in chronic DM seriously affect the outcome of the long-term complications caused by oxidative stress. Antioxidants have been reported to reduce the complications in DM by arresting free radical damage. ${ }^{9}$ As reflected in their structural similarity, tocopherol and tocotrienol are well recognized for their antioxidant effects. ${ }^{10}$ The tocotrienolrich fraction (TRF) from palm oil sources is a rich source of vitamin $\mathrm{E}$, which consists of a majority of tocotrienol and a smaller proportion of tocopherol. The TRF is an excellent antioxidant, and it has been effectively used as a nutritional supplement due to its potential therapeutic benefits. ${ }^{11}$

The present study was undertaken to observe the effects of palm oil TRF on plasma glucose, lipid levels, oxidative stress and histological changes in the thoracic aortas of STZ-induced diabetic rats. While the fatty acid composition of palm oil may have a weak cholesterol-raising potential, it may also have beneficial effects on the atherosclerotic process in DM. We believe that valuable information regarding the protective effects of TRF in DM will be helpful in treating the oxidative damage caused by DM.

\section{MATERIALS AND METHODS}

\section{Experimental groups and diabetes induction}

Male Sprague Dawley rats weighing 260-290 gm each were obtained from the Animal Unit of Universiti Kebangsaan Malaysia. Two animals were housed in each cage. All animals were maintained on a balanced diet and water ad libitum. The study was approved by Universiti Kebangsaan Malaysia Animal Ethics Committee Animal Ethics Committee (UKMAEC).

Animals were divided into three groups: (i) normal non-diabetic (NDM), (ii) diabetic treated (TRF) and (iii) diabetic untreated (non-TRF). Diabetes was induced in the diabetic group after overnight fasting of the rats, followed by single intravenous injection (via the tail vein) of STZ (Sigma, St Louis, MO, USA), which was freshly dissolved in normal saline. The dose of STZ was $50 \mathrm{mg} / \mathrm{kg}$ body weight. The NDM group, which consisted of seven rats $(n=7)$, was infused with normal saline. Three days later, blood was collected via the tail vein, and glucose concentrations were measured by a strip-operated blood glucose sensor (Companion 2, Medisense Ltd., Birmingham, UK). Rats with blood glucose levels $>15.0 \mathrm{mmol} / \mathrm{l}$ were included in the study and further subdivided in two groups: a group supplemented with TRF (TRF: $\mathrm{n}=8$ ) and a group not supplemented with TRF (non-TRF: $n=8$ ). TRF was obtained from the Malaysian Palm Oil Board (MPOB) and was administered orally at a dose of $200 \mathrm{mg} / \mathrm{kg}$ body weight /day. TRF was administered every day, over a feeding period of eight weeks. The nonTRF and NDM groups were left untreated.

\section{Biochemical investigations}

After eight weeks of supplementation, the rats were fasted overnight, and blood was collected by cardiac puncture under deep anesthesia with diethyl ether. Blood was collected into tubes containing sodium fluoride (for fasting blood glucose (FBG) analysis) and EDTA. The upper third of the thoracic aorta was taken for electron microscopic examination and the rest was used for biochemical investigations. Aortic homogenates were prepared essentially as described by Upston et al. ${ }^{12}$ Blood was kept on ice and centrifuged at $3000 \mathrm{rpm}$ for $20 \mathrm{~min}$ at $4{ }^{\circ} \mathrm{C}$; the plasma obtained was stored at $-40^{\circ} \mathrm{C}$ until analysis.

Plasma glucose levels were analyzed using enzymatic glucose-oxidase kits (Trace Scientific, Melbourne, Australia. Catalogue no TR 15104). Blood HbA1c levels were determined using a method described by Eross et al. and were expressed as a percentage of total hemoglobin. ${ }^{13}$ Determinations of total cholesterol (TC), triglyceride and high density lipoprotein (HDL-C) levels were carried out using kits (Teco Diagnostics, 1286 N. Lakeview Ave., Anaheim). Low-density lipoprotein (LDL-C) concentrations were determined using a method described by Friedewald et al. ${ }^{14}$

Protein concentrations were measured with the Bradford assay. ${ }^{15}$ MDA+HNE levels were assayed by lipid peroxidation, using a kit (Calbiochem's Lipid Peroxidation Assay, catalogue No. 437634). SOD activities were determined according to Beyer and Fridovich. ${ }^{16}$ According to this method, SOD activities were measured by photochemical nitro blue tetrazolium (NBT) reduction (one unit of SOD is the amount that causes a 50\% decrease in NBT reduction, due to SOD-mediated inhibition).

Plasma vitamin $\mathrm{C}$ levels were measured according to Liau et al. ${ }^{17}$ Briefly, $100 \mu \mathrm{l}$ of plasma was mixed with 100 $\mu \mathrm{l}$ of $10 \%$ perchloric acid containing $1 \%$ meta-phosphoric 
acid. These were mixed in 1.5-ml Eppendorf tubes, wrapped in aluminum foil to ensure that the vitamin $\mathrm{C}$ would not be degraded by light, and kept at $-40{ }^{\circ} \mathrm{C}$. Vitamin $\mathrm{C}$ analysis was performed within one week of storage, using highperformance liquid chromatography (HPLC) at room temperature. The HPLC system consisted of a Gilson 307 pump, a Rheodyne manual injector, a 20- $\mu$ l sample loop and a Gilson 151 UV/VIS set at $245 \mathrm{~nm}$. The column used for separation was a Metaphase Crestpak C18S (4.6 mm I.D. x $150 \mathrm{~mm} \mathrm{~L}$ ). The mobile phase consisted of $0.015 \%$ metaphosphoric acid in $20 \mathrm{mM}$ ammonium dihydrogen phosphate ( $\mathrm{pH}$ 2.95). The flow rate was set at $1 \mathrm{ml} / \mathrm{min}$ with a run time of 10 minutes. The eluted peak of vitamin $\mathrm{C}$ was detected using a UV/VIS Gilson set at $245 \mathrm{~nm}$, and results were analyzed based on the area under the peak.

Prior to injection of the sample into the system, $200 \mu \mathrm{l}$ of HPLC mobile phase was added to the sample. Samples were then vortexed and centrifuged at 20,000 $g$ for 1 minute. A total of $10 \mu \mathrm{l}$ of supernatant was then injected into the HPLC for vitamin $\mathrm{C}$ analysis. We produced a standard curve daily in order to extrapolate the true concentration of vitamin $\mathrm{C}$ in the sample. The standard curve was determined by diluting $10 \mathrm{mg}$ of L-(+)-ascorbic acid (BDH, Poole, UK) in $10 \mathrm{ml}$ of fresh mobile phase. The mixed solution was then diluted further with $1 \%$ metaphosphoric acid and $10 \%$ perchloric acid, to produce a stock solution with a concentration of $100 \mathrm{mg} / \mathrm{ml}$. External control of analysis was performed by injecting a vitamin $\mathrm{C}$ standard for every 10 injections. An external control concentration deviation of $5 \%$ resulted in the creation of a new standard curve.

The Alkaline Comet Assay procedure was performed according to the method described by Singh et al. ${ }^{18}$ Electrophoresis slide observation was performed by using a Leitz Laborlux fluoresence microscope (Nikon) equipped with an epifluorescence mercury lamp source (excitation filter $515 \mathrm{~nm}$, barrier filter $590 \mathrm{~nm}$ ) and an X40 fluorescence objective (numerical aperture 0.85 ). Data were analyzed using a specialized single-cell gel (SCG) image analysis program (TriTex Comet Score ${ }^{\mathrm{TM}}$ ).

The image analyzer software provided a full range of densitometric and geometric parameters describing the complete comet, as well as the head and tail of the DNA portions. Since the comet assay essentially reflects the displacement of fluorescence from the head to the tail in damaged cells, the results were expressed as percentages of comet tail intensity (fraction of DNA in the tail divided by the amount of DNA in the cell, multiplied by $100-\%$ tail). The comet tail moment (product of the tail and the mean distance of migration in the tail [arbitrary units]) was selected as the parameter to quantify basal levels of DNA damage. Each slide was analyzed in duplicate, and 50 cells per slide were scored.

\section{Transmission electron microscopic evaluation}

The microscopic evaluation of the aorta was performed according to the protocol described by Karasu et al. ${ }^{19}$ Incidentally, Karasu et al. ${ }^{17}$ also used three groups for their study: NDM, untreated diabetic and vitamin-E-treated groups. ${ }^{19}$ Following dissection, the thoracic aorta was cut into small pieces, fixed at $4^{\circ} \mathrm{C}$ for $2 \mathrm{hr}$ in $2.5 \%$ buffered glutaraldehyde and post-fixed for $1.5 \mathrm{hr}$ with osmium tetraoxide. Tissues were dehydrated in ethyl alcohol, followed by propylene oxide, and were embedded in araldite. Semi-thin sections of plastic-embedded samples were stained with toluidine blue. Ultrathin circumferential sections were stained with uranyl acetate and lead citrate and were subsequently examined by a Tecnai G2 Transmission Electron Microscope (FEI, USA).

\section{Statistical analysis}

All results were expressed as the mean \pm SEM. The data were analyzed by one-way analysis of variance (ANOVA), followed by a post-hoc LSD multiple comparison test, to estimate the significance of the differences between the groups. The differences between the groups were considered to be significant if $\mathrm{p}<0.05$.

\section{RESULTS}

The food and water intakes of the non-TRF diabetic group were markedly increased as compared to those of the NDM group (Figures 1A and 1B). The food and water intakes, although expressed per rat, were calculated per cage, so that the statistical results would not be influenced. TRF supplementation had a considerable effect on water and food consumption throughout the study. At the end of the study period, both non-TRF and TRF groups exhibited a significant loss of body weight when compared to the NDM group. However, TRF supplementation prevented further loss of body weight. The loss of body weight in TRF-supplemented rats was significantly lower than that of the non-TRF group (Figure 2).

FBG, HbA1c, TC, LDL-C, HDL-C and triglyceride levels for all groups are shown in table 1. FBG and HbA1c levels were found to be higher in the non-TRF and TRF groups than in the NDM group. However, significant reductions in FBG and $\mathrm{HbA1c}$ levels were observed in the TRF group, as compared to the non-TRF group. Plasma TC, LDL-C and triglyceride concentrations were markedly increased in non-TRF diabetic rats, as compared to the NDM 

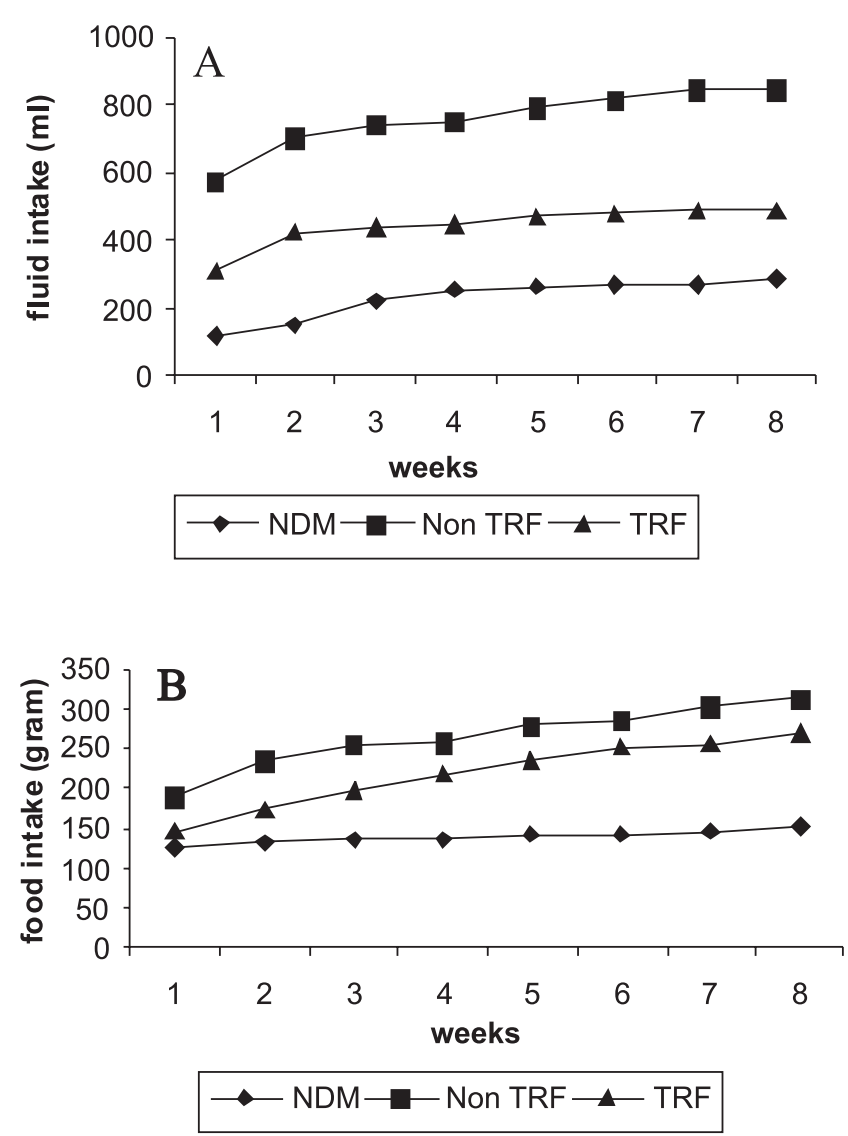

Figure 1 - Mean weekly water (A) and food (B) intakes in different groups of rats. Food and water intakes were calculated per cage (two animals in a cage), so statistical analysis was not possible

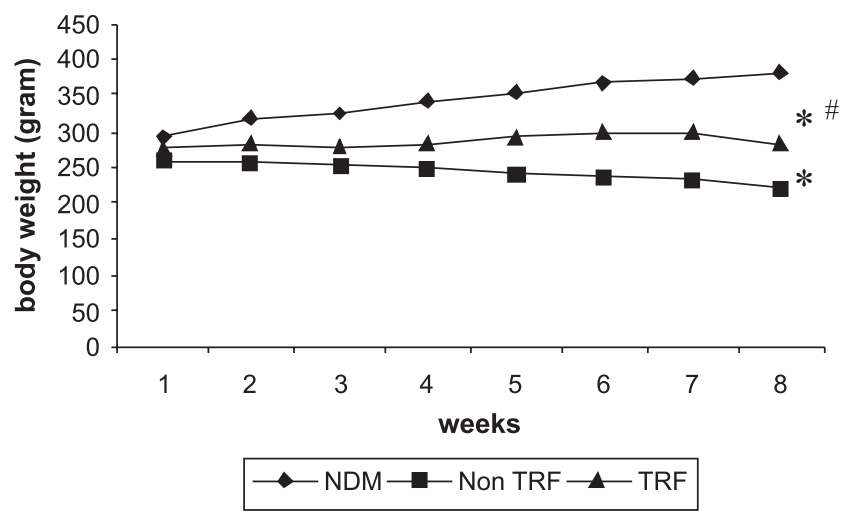

Figure 2 - Rat body weight in different groups of study. $* \mathrm{p}<0.05$ vs. NDM group, ${ }^{*} \mathrm{p}<0.05$ vs. non-TRF group

rats. TRF supplementation prevented the increase of TC, LDL-C and triglyceride concentrations, where these levels were significantly lower than those of the non-TRF rats. On the other hand, the TRF supplementation group also had significantly higher levels of HDL-C, as compared to the non-TRF group.

The oxidative stress parameters in plasma and aortic homogenates are shown in table 2. STZ-induced diabetes resulted in a significant decrease in plasma and aortic homogenate SOD activities, as well as in plasma vitamin C levels. However, plasma SOD activities of the TRF group were significantly higher than those of the non-TRF group.

Table 1 - Plasma glucose, HbA1c and lipid profiles in NDM, non-TRF and TRF groups following eight weeks of study

\begin{tabular}{lccc}
\hline Parameter & \multicolumn{2}{c}{ Groups } \\
\cline { 2 - 4 } & NDM group & Non-TRF group & TRF group \\
\hline FBG (mmol/l) & $6.90 \pm 0.22$ & $31.22 \pm 0.15 *$ & $26.23 \pm 0.65 * \#$ \\
HbAlc $(\%)$ & $4.57 \pm 0.28$ & $10.95 \pm 0.93 *$ & $8.64 \pm 0.31 * \#$ \\
TC $(\mathrm{mg} / \mathrm{dl})$ & $45.59 \pm 2.44$ & $72.11 \pm 3.50 *$ & $53.38 \pm 3.28 \#$ \\
Triglycerides (mg/dl) & $37.64 \pm 1.08$ & $149.35 \pm 11.19 *$ & $105.73 \pm 4.84 * \#$ \\
LDL-C (mg/dl) & $18.93 \pm 1.01$ & $38.94 \pm 3.87 *$ & $14.53 \pm 1.18 * \#$ \\
HDL-C (mg/dl) & $20.84 \pm 1.59$ & $4.16 \pm 0.58 *$ & $17.67 \pm 1.27 * \#$ \\
\hline P & &
\end{tabular}

Plasma glucose, HbA1c and lipid profiles in NDM, non-TRF and TRF groups.

Table 2 - MDA+HNE and SOD in plasma and aorta, plasma vitamin C levels and DNA damage in peripheral lymphocytes in NDM, non-TRF and TRF groups following eight weeks of study

\begin{tabular}{lccc}
\hline Parameter & \multicolumn{3}{c}{ Groups } \\
\cline { 2 - 3 } & NDM group & non-TRF group & TRF group \\
\hline MDA+HNE plasma (nmol/g protein) & $14.51 \pm 1.44$ & $25.00 \pm 1.08^{*}$ & $11.11 \pm 0.54^{\#}$ \\
MDA+HNE aorta ( $\mu$ mol/g protein) & $10.71 \pm 1.15$ & $14.79 \pm 1.46^{*}$ & $5.34 \pm 0.60^{\#}$ \\
SOD plasma (u.e/g protein) & $3.33 \pm 0.14$ & $1.27 \pm 0.11^{*}$ & $1.97 \pm 0.12 * *$ \\
SOD aorta (u.e/g protein) & $3.19 \pm 0.06 \times 10^{2}$ & $1.50 \pm 0.19 \times 10^{2 *}$ & $1.06 \pm 0.06 \times 10^{2 *}$ \\
Vit. C plasma $(\mu \mathrm{g} / \mathrm{ml})$ & $1.17 \pm 0.08$ & $0.66 \pm 0.08^{*}$ & $2.42 \pm 0.06^{\#}$ \\
Comet tail intensity $(\%)$ & $4.00 \pm 2.46$ & $7.11 \pm 0.35^{*}$ & $5.59 \pm 0.51^{\#}$ \\
Comet tail moment (arbitrary unit) & $0.46 \pm 0.04$ & $5.76 \pm 0.38^{*}$ & $0.32 \pm 0.04^{* *}$ \\
\hline Da
\end{tabular}

Data are represented as the mean \pm SEM. ${ }^{*} \mathrm{p}<0.05$ compared with NDM group, ${ }^{*} \mathrm{p}<0.05$ compared with No Suppl group. 
TRF supplementation also inhibited the reduction of plasma vitamin $\mathrm{C}$ in diabetic rats. MDA+HNE levels of plasma and aortic homogenates were significantly increased in the non-TRF diabetic groups, as compared to the NDM group. Interestingly, treatment of the diabetic rats with TRF also normalized the plasma and aortic homogenate MDA $+\mathrm{HNE}$ levels. The levels of endogenous DNA damage in the lymphocytes (comet tail intensity and comet tail moment) were significantly higher in the non-TRF rats than in the NDM rats. TRF supplementation prevented DNA damage.

\section{Morphological observation of the aorta}

Under electron microscopic examination, the aortas from the control rats were observed to have smooth muscle cells, internal elastic lamina and a small amount of extracellular matrix between the elastic lamina and vascular smooth muscle cells (VSMC) (Figure 3A). The aortic tunica media also showed a regular appearance, including smooth muscle cells, between the elastic lamina and within a homogenous interstitial matrix. Endothelial cells in the aortas from the control rats demonstrated a characteristic smooth and uniform phenotype (Figure 3B).

STZ-induced diabetes caused severe alterations in the structure of the vascular wall in non-TRF rats, as shown in Fig. 4A, 4B and 4C. The tunica intima of the aorta was irregular and the endothelial cells showed irregular distributions with atrophic characteristics. The subendothelial region was thickened in patches; infiltrating mononuclear cells were observed. Aortic media showed irregular VSMCs between partly fragmented elastic lamina within degenerative interstitial matrix. The elastic lamina also appeared fragmented and reduplicated. Irregularly fragmented and atrophic smooth muscle cells were frequently observed together with excess extracellular matrix, predominantly consisting of electron-dense amorphous material. There was a migration of VSMC from the tunica media into the tunica intima, with a break in the elastic lamina.

TRF treatment of diabetic rats reduced VSMC proliferation and degeneration and also inhibited the formation of electron-dense amorphous material in the aortic media. The elastic lamina was more regular, and the intima surface appeared smoother, with fewer defects. Endothelial cells of the aortas from TRF-treated rats still exhibited squamous characteristics (Figure 5).

\section{DISCUSSION}

Administration of STZ to rats destroyed pancreatic $\beta$-cells, leading to inhibited insulin secretion, thereby increasing plasma glucose levels. The diabetic rats also exhibited increased food intake, due to the inability of cells to obtain glucose as an energy source. This may have caused the increase in blood glucose levels. Consequently, the diabetic rats obtained their energy from the catabolism of protein and fat, which may eventually lead to a reduction in body weight. Hyperglycemia also leads to polyuria and polydipsia.

An earlier protocol reported by Karasu et al. (1997) was followed and vitamin E (200 mg/kg body weight) was administered for eight weeks. ${ }^{19}$ The food and fluid intake, and the body weight were measured according to a protocol
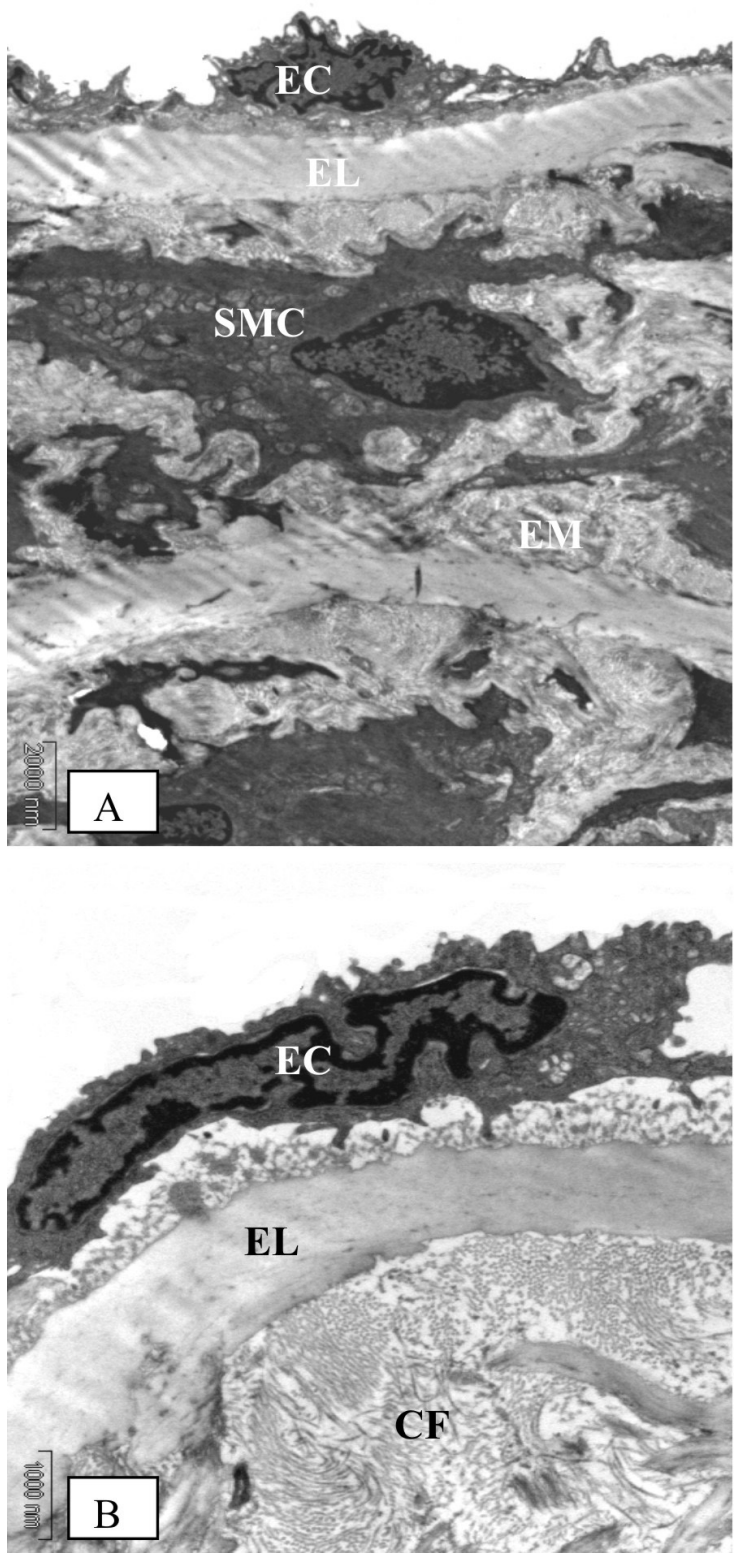

Figure 3 - Electron microscopic findings on the aorta of control rats at eight weeks of study. (A) (Original magnification $x$ 4400) The media layer is composed mainly of smooth muscle cells (SMC) with normal appearance, elastic lamina (EL) and extracellular matrix (EM) between the elastic lamina and smooth muscle cells. (B) (Original magnification x 11000) Extracellular matrix contains scattered collagen fibers (CF) and the endothelial cells appear smooth 

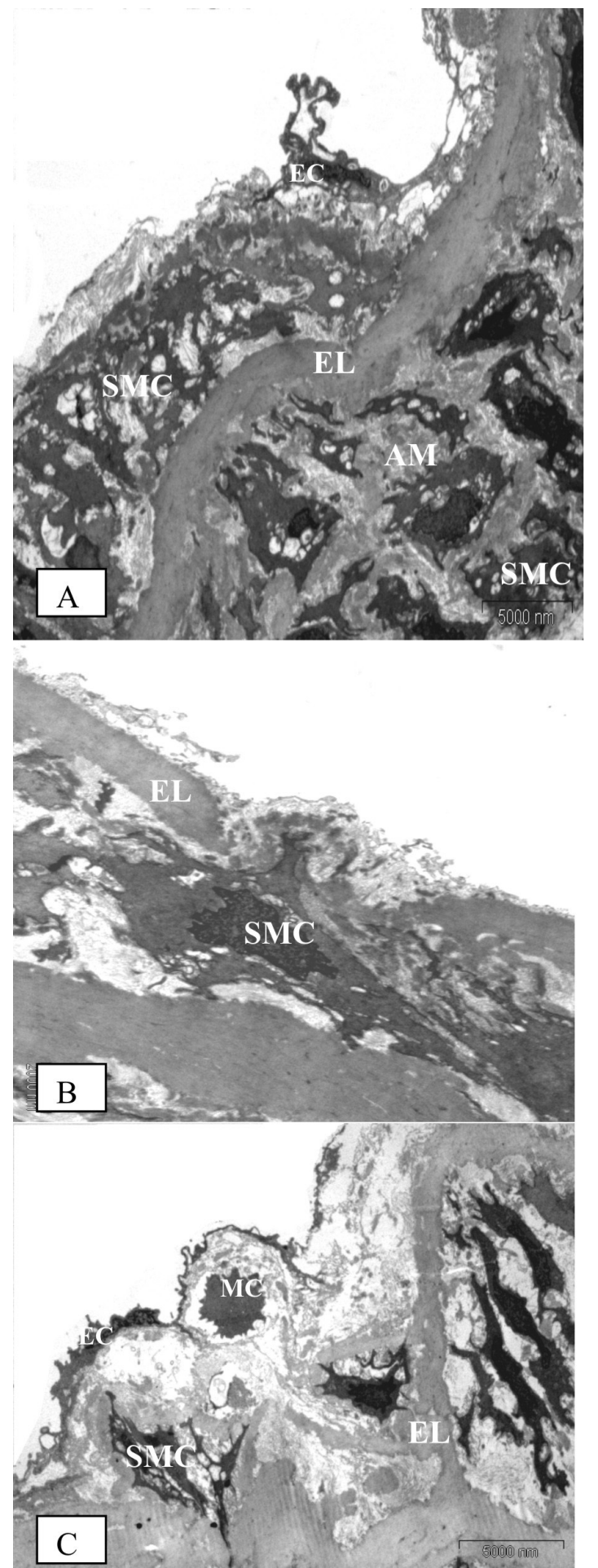

Figure 4 - Electron microscopic findings on the aorta of the diabetic rats following eight weeks of study. (A) - (Original magnification $x$ 4400) Proliferation and fragmentation of medial smooth muscle cells (SMC) in both the intima and media layers. The extracellular matrix also consists predominantly of amorphous material (AM), as apparent in the media of diabetic rats. Endothelial cells (EC) lost their squamous characteristics and became atrophic. (B) - (Original magnification x 4400) A smooth muscle cell (SMC) is migrating from the media into the intima, breaking through the elastic lamina (EL). (C) - (Original magnification x 6500) A mononuclear cell (MC) and fragmented smooth muscle cell (SMC) at the subendothelial layer of the intima. We observed fragmentation and reduplication of elastic lamina

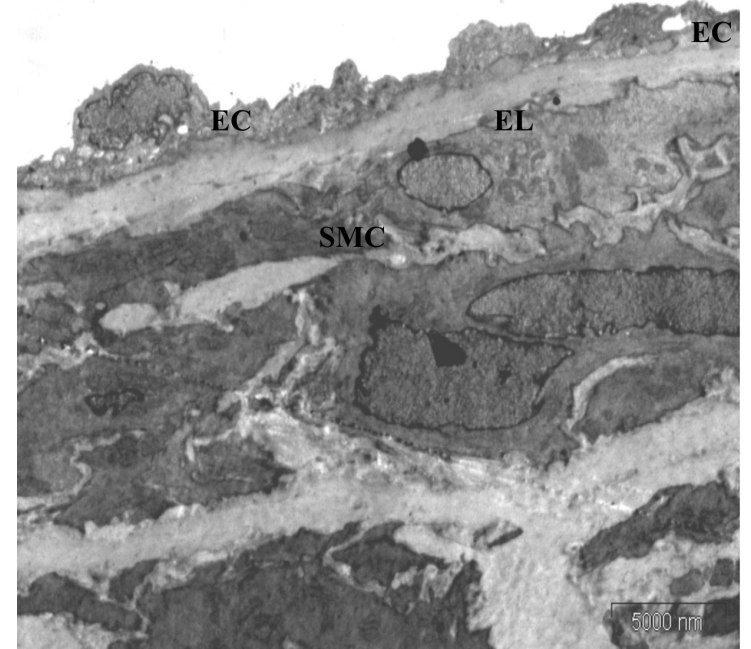

Figure 5 - (Original magnification $\mathrm{x} 4400$ ) Electron microscopic findings on the aorta of TRF group following eight weeks of study. The elastic lamina (EL) appeared more regular, and smooth muscle cells (SMC) did not actively proliferate. The endothelial cells (EC) still display squamous characteristics

proposed by Kocak et al. ${ }^{20}$ An earlier study by Kocak et al. involved three animals per cage, whereas we had only two animals per cage. Therefore, a statistical analysis was not possible. $^{20}$

Our study showed higher levels of glycemic status and food and fluid intake as well as decreased body weight, which had been reported in the past. ${ }^{20}$ After eight weeks of TRF supplementation, the glycemic status in STZ-induced diabetic rats was significantly improved by reducing the levels of FBG and HbA1c. The improved glycemic status may be the main cause of the slowing of body weight loss in the context of decreased food and fluid intake in the TRFsupplemented rats.

Vitamin E supplementation has been shown to decrease $\mathrm{HbA} 1 \mathrm{c}$ and lipid peroxidation levels in type 1 diabetic patients. ${ }^{21}$ It was also suggested that the improvement of glycemic status could be related to an improvement in glucose metabolism. ${ }^{21}$ Supplementation of vitamin E for four weeks prevented hyperglycemia due to fish oil intake in healthy volunteers. ${ }^{22}$ Vitamin E supplementation could also increase the production of insulin and increase insulin availability: the glucose ratio and the antioxidant effects of vitamin $\mathrm{E}$ may have prevented $\beta$-cell destruction, which occurred due to the lipid peroxidation process. ${ }^{22}$

Vitamin E supplementation prior to the induction of diabetes by STZ prevented the destruction of pancreatic $\beta$-cells and also improved the glycemic status in diabetic rats. ${ }^{23}$ TRF supplementation in our study began on the third day following diabetic induction. Although the destruction of $\beta$-cells caused chronic hyperglycemia, further destruction 
may have been prevented by TRF, probably through an antioxidant mechanism.

The increase in glycemic status could produce a rise in the values of lipid parameters, as shown in the non-TRF group. The increase in TC, LDL-C and triglyceride levels and the decrease in HDL-C confirmed the dyslipidemia pattern in type 1 diabetes mellitus, in agreement with a previous study. ${ }^{24}$ In another research study, HbAlc levels were noted as having a positive correlation with TC, LDL-C, triglycerides and VLDL-C in diabetes mellitus patients. ${ }^{25}$ Thus, the results of our study supported the relationship of poor glycemic control and higher risk of cardiovascular complications due to a rise in plasma concentrations of TC, triglycerides and LDL-C as well as low concentrations of HDL-C. Based on the results, we postulate that metabolic control of this disease is directly related to the severity of the atherogenic process. In this study, diabetic rats supplemented with TRF exhibited lower levels of TC, LDL-C and triglyceride and increased levels of HDL-C. Therefore, by improving lipid metabolism, TRF prevented the pathogenesis of cardiovascular complications involved in DM.

Free radicals are considered to underlie the pathogenesis of DM and its complications. The impact of free radicals on lipids is denoted as lipid peroxidation; it was found that plasma and tissue lipid peroxidation products were much more abundant in diabetic conditions. ${ }^{6}$ Free radicals including hydroxyl radicals, singlet oxygen, peroxyl radicals and peroxynitrite were able to produce other modifications to DNA bases, as well as causing strand breakage and other types of DNA damage. ${ }^{26}$ Hyperglycemia causes increased production of free radicals and insufficiencies in the antioxidant system, thus increasing oxidative stress. ${ }^{19}$ In accordance with previous findings, our results show that the levels of lipid peroxidation products such as MDA+HNE, as well as the levels of DNA damage in lymphocytes, were significantly higher in the non-TRF group, as compared to the NDM group.

The efficacy of antioxidants in reducing oxidative stress in DM has been proven by researchers. Carvedilol is a non-selective beta-adrenoceptor and a selective alphaadrenoceptor antagonist that has antioxidant and free radical scavenger properties; the drug was able to protect diabetic rats by reducing oxidative stress. ${ }^{27}$ Alpha lipoic acid, a universal antioxidant, has been proven to reduce oxidative stress in diabetic rats and significantly improves diabetic neuropathy. ${ }^{28}$ In addition, antioxidants such as vitamin C, trolox and prubacol also prevented the STZ-induced elevation of DNA damage in the liver and kidney that is typical of diabetic rats. ${ }^{29}$

The antioxidant activities of tocopherols and tocotrienols are rooted in their ability to donate phenolic hydrogens (electrons) to lipid radicals. ${ }^{10}$ Tocotrienols also have greater antioxidant activity than tocopherols and are more efficient protectors against some free radical-related diseases than tocopherols. ${ }^{10,30}$ TRF has been used as a nutritional supplement; it has substantial therapeutic potential and is capable of scavenging free radicals through its antioxidant

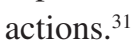

In the present study, TRF supplementation prevented an increase in MDA + HNE levels and inhibited DNA damage in diabetic rats. This finding indicates that TRF has potential in reducing oxidative damage. The percentage of comet tail intensity in the TRF group was significantly reduced when compared to that of the untreated diabetic rats, but was markedly higher when compared to that of the NDM group. Furthermore, the levels of DNA damage, as measured by the comet tail moment, showed no significant difference when compared between the NDM and TRF groups. This result suggests that the DNA damage that occurs in TRF-supplemented rats had been repaired. Our study also demonstrates that TRF increases the levels of vitamin $\mathrm{C}$ and $\mathrm{SOD}$ activity. The elevated vitamin $\mathrm{C}$ levels, increased SOD activity and reduced lipid peroxidation and DNA damage in the TRF-supplemented group suggest that TRF plays a protective role against oxidative stress in diabetic conditions. These observations show that TRF may improve the antioxidant defense mechanism by decreasing free radical levels and thereby reducing the oxidative damage that occurs in diabetic conditions.

Our results are consistent with those of a previous study in which it was reported that tocotrienols from palm oil act as potent inhibitors of lipid peroxidation and protein oxidation. ${ }^{32}$ In addition, TRF that was isolated from rice bran oil also exhibited hypolipidemic and antioxidant properties in experimentally induced hyperlipidemic rats. ${ }^{33}$ TRF exhibited powerful antioxidant properties in protecting murine skin from oxidative damage induced by UV-irradiation. ${ }^{34} \mathrm{TRF}$ has also shown potential in enhancing the process of wound healing in diabetic animals, perhaps by reducing the levels of oxidative stress markers. ${ }^{35}$

The morphological findings of this study showed that the ultrastructural organization of the aorta is damaged in STZ-induced diabetic rats. Various morphological changes in endothelial cells are indicative of endothelial injury. Infiltration of macrophages into the subendothelial space, migration of medial smooth muscle cells to the intima layer and proliferation of medial smooth muscle cells in the intima layer were observed in diabetic rats. These findings are considered to be early events in the development of atherosclerotic lesions. ${ }^{36}$ The increase in MDA+HNE levels and decrease in SOD activity in thoracic aortas reflect increased oxidative stress. The elevation of MDA in the 
aorta is due to increased production and liberation of lipid peroxides that result from pathological changes. ${ }^{37}$

In line with past studies, our results also demonstrated that hyperglycemia and hyperlipidemia are associated with morphological alterations in the aorta and could be important factors in the initiation of atherosclerotic lesion development in STZ-diabetic rats. ${ }^{19}$ Furthermore, high oxidative stress also plays an important role in the development of diabetes complications. ${ }^{38,39}$ It has also been stated that diabetic cardiovascular complications are directly related to conditions of high oxidative stress. ${ }^{6}$

This study demonstrated that eight weeks of supplementation with TRF resulted in reduced damage to the aortic wall. The mechanism by which TRF supplementation affects vascular morphology involves its antioxidant properties; supplementation with TRF effectively prevents an increase in MDA + HNE levels in the thoracic aortas of diabetic rats. It has been reported that vitamin E prevents the proliferation of VSMC in diabetes, and this effect may be mediated through an enhancement of free radical scavenging. ${ }^{40}$ The effect of vitamin E on VSMC proliferation would also be mediated through its lipid-lowering action..$^{40}$ In addition, TRF is proven to be a more effective inhibitor of in vitro LDL oxidation and endothelial cell lipid peroxidation than alpha tocopherol. ${ }^{41}$ This would suggest that reducing oxidative stress leads to an improvement in the morphological changes of the aortic wall in diabetic rats.

The effect of TRF on vascular morphology is also attributed to its effects on carbohydrate and lipid metabolism. In the present study, the treatment of diabetic rats with TRF effectively inhibited dyslipidemia and improved hyperglycemia. This reduction in plasma lipids would contribute to a decrease in circulating oxidized LDL, thereby preventing endothelial injury. Our findings are in line with previous reports in which it was shown that TRF reduced TC, triglyceride and LDL-C levels and inhibited the progression of atherosclerosis in rabbits fed an atherogenic diet. ${ }^{42}$ Furthermore, it was also demonstrated that VSMC proliferation induced by hyperglycemia could be preserved after the normalization of the glucose concentration, suggesting that it is very important to prevent the process of atherosclerosis in DM patients while maintaining good glycemic control. ${ }^{40}$

Palm oil contains mainly tocotrienols, as well as other vitamin E supplements such as tocopherols. The synergistic actions of both tocotrienols and tocopherols may contribute to the protective actions of palm oil. In this study, both components may have contributed to the beneficial effects. Larger clinical trials may be needed to demonstrate the beneficial effects of palm oil in combating oxidative stress involved in diseases such as DM and atherosclerosis.

\section{CONCLUSION}

The present findings show that palm oil TRF improved blood glucose, dyslipidemia and oxidative stress in diabetic rats. Furthermore, hypoglycemic, hypolipidemic and antioxidative stress effects of TRF may be associated with the prevention of development of abnormalities in the structure of thoracic aortas in diabetic rats. Therefore, we suggest that palm oil TRF is able to prevent the progression of vascular wall changes occurring in DM. However, further studies may be needed to elucidate this phenomenon.

\section{ACKNOWLEDGMENTS}

The authors would like to express their gratitude to Malaysian Ministry of Science and Technology for the funding this research under research grant no: 05-01-02SF0014. We would also like to thank Mr. Abdul Gapor Mohd Top from Malaysian Palm Oil Board for supplying us the Palm oil Tocotrienol Rich Fraction that had been used in this research.

\section{REFERENCES}

1. Wild S, Roglic G, Green A, Sicree R, King H. Global prevalence of diabetes: estimates for the year 2000 and projections for 2030. Diabetes Care. 2004;27:1047-53.

2. Klein R. Hyperglycemia and microvascular and macrovascular disease in diabetes. Kelly West Lecture. Diabetes Care. 1995;18:258-68.

3. Gu K, Cowie CC, Harris MI. Mortality in adults with and without diabetes in a national cohort of the U.S. population, 1971-1993. Diabetes Care. 1998;21:1138-45.
4. Rösen P, Nawroth PP, King G, Möller W, Tritschler HJ, Packer L. The role of oxidative stress in the onset and progression of diabetes and its complications: a summary of a Congress Series sponsored by UNESCOMCBN, the American Diabetes Association and the German Diabetes Society. Diabetes Metab Res Rev. 2001;17:189-212.

5. Halliwell B, Whiteman M. Measuring reactive species and oxidative damage in vivo and in cell culture: how should you do it and what do the results mean? Br J Pharmacol. 2004;142:231-55. 
6. Jay D, Hitomi H, Griendling KK. Oxidative stress and diabetic cardiovascular complications. Free Radic Biol Med. 2006;40:183-92.

7. Abou-Seif MA, Youssef AA. Evaluation of some biochemical changes in diabetic patients. Clin. Chim Acta. 2004;346:161-70.

8. Price KD, Price CS, Reynolds RD. Hyperglycemia-induced ascorbic acid deficiency promotes endothelial dysfunction and the development of atherosclerosis. Atherosclerosis. 2001;158:1-12.

9. Peerapatdit T, Likidlilid A, Patchanans N, Somkasetrin A. Antioxidant status and lipid peroxidation end products in patients of type 1 diabetes mellitus. J Med Assoc Thai. 2006;89 Suppl 5:S141-46.

10. Kamal-Eldin A, Appelqvist LA. The chemistry and antioxidant properties of tocopherols and tocotrienols. Lipids. 1996;31:671-701.

11. Theriault A, Chao JT, Wang Q, Gapor A, Adeli K. Tocotrienol: a review of its therapeutic potential. Clin Biochem. 1999;32:309-19.

12. Upston JM, Witting PK, Brown AJ, Stocker R, Keany JF Jr. Effect of vitamin $\mathrm{E}$ on aortic lipid oxidation and intimal proliferation after arterial injury in cholesterol-fed rabbits. Free Radic Biol Med. 2001;31:124553.

13. Eross J, Kreutzmann D, Jimenez M, Keen R, Rogers S, Cowell C, et al. Colometric measurement of glycosylated protein in whole blood, red blood cells, plasma and dried blood. Ann Clin Biochem. 1984;21:47783.

14. Friedewald WT, Levy RI, Fredickson DS. Estimation of the concentration of low- density lipoprotein cholesterol in plasma without use of the preparative ultracentrifuge. Clin Chem. 1972;18:499-502.

15. Bradford MM. A rapid and sensitive method for the quantitation of microgram quantities of protein utilizing the principle of protein-dye binding. Anal Biochem.1976;72:248-54.

16. Beyer WF Jr, Fridovich I. Assaying for superoxide dismutase activity: some large consequences of minor changes in conditions. Anal Biochem. 1987;161:559-66.

17. Liau LS, Lee BL, New AL, Ong CN. Determination of plasma ascorbic acid by high-performance liquid chromatography with ultraviolet and electrochemical detection. J Chromatogr.1993; 612: 63-70.

18. Singh NP, Mccoy T, Tice RR., Schneider EL. A simple technique for quantitation of low levels of DNA damage in individual cells. Exp Cell Res. 1988;175:184-91.

19. Karasu C, Ozansoy G, Bozkurt O, Erdo an D, Omero lus S. Antioxidant and Triglyceride-lowering effects of vitamin $\mathrm{E}$ associated with the prevention of abnormalities in the reactivity and morphology of aorta from streptozotocin-diabetic rats. Antioxidants in Diabetes-Induced Complications (ADIC) Study Group. Metabolism. 1997;46:872-79.

20. Kocak G, Aftan F, Elberg S, Yildizoglu-Ari N, Karasu C. Alpha-lipoic acid treatment ameliorates metabolic parameters, blood pressure, vascular reactivity and morphology of vassel alredy damaged by streptozotocin-diabetic rats. Diab Nutr Metab. 2000;13:308-18.

21. Jakus V, Bauerova K, Michalkova D, Carsky J. Value markers of advanced glycation and lipoxidation in serum proteins of children with diabetes mellitus. Bratisl Lek Listy. 2000;101:484-89.

22. Luostarinen R, Wallin R, Wibel L, Saldeen T. Vitamin E supplementation counteracts the fish oil-induced increase of blood glucose in humans. Nutr Res. 1995;15:953-68.
23. Ihara Y, Yamada Y, Toyokuni S, Miyawaki K, Ban N, Adachi T, Kuroe A, Iwakura T, Kubota A, Hiai H, Seino Y. Antioxidant alpha-tocopherol ameliorates glycemic control of GK rats, a model of type 2 diabetes. FEBS Lett. 2000; 473:24-6.

24. Hayashibe H, Asayama K, Nakane T, Kobayashi K, Amemiya S, Nakazawa S. Decreased activity of plasma cholesteryl ester transfer protein in children and adolescents with insulin-dependent diabetes mellitus. Acta Paediatr.1999;88:1067-70.

25. Ohta T, Nishiyama S, Nakamura T, Saku K, Maung KK, Matsuda I. Predominance of large low density lipoprotein particles and lower fractional esterification rate of cholesterol in high density lipoprotein in children with insulin-dependent diabetes mellitus. Eur J Pediatr. 1998; $157: 276-81$

26. Imlay JA, Linn S. DNA damage and oxygen radical toxicity. Science. 1998;240:1302-09.

27. Huang H, Shan J, Pan XH, Wang HP, Qian LB. Carvedilol protected diabetic rat hearts via reducing oxidative stress. J Zhejiang Univ Sci B. 2006; 7:725-33

28. Packer L, Kraemer K, Rimbach G. Molecular aspects of lipoic acid in the prevention of diabetes complications. Nutr. 2001;17:888-95

29. Imaeda A, Kaneko T, Aoki T, Kondo Y, Nagase H. The DNA damage and the effect of antioxidant in streptozotocin-treated mice. Food and Chemical Toxicology. 2002;40: 979-87.

30. Suarna C, Hood RL, Dean RT, Stocker R. Comparative antioxidant activity of tocotrienols and other natural lipid-soluble antioxidants in a homogeneous system, and in rat and human lipoproteins. Biochim Biophys Acta. 1993;1166:163-70.

31. Serbinova E, Khwaja S, Catudioc J, Ericson J, Torres Z, Gapor A, Kagan V, Packer L. Palm oil vitamin E protects against ischemia/ reperfusion injury in the isolated perfused langendorff heart. Nutr. Res. Suppl. 1992;12:S203-S15.

32. Kamat JP, Devasagayam TP. Tocotrienols from palm oil as potent inhibitors of lipid peroxidation and protein oxidation in rat brain mitochondria. Neurosci Lett. 1995;195:179-82.

33. Minhajuddin M, Beg ZH, Iqbal J. Hypolipidemic and antioxidant properties of tocotrienol rich fraction isolated from rice bran oil in experimentally induced hyperlipidemic rats. Food Chem. Toxicol. 2005; 43:747-53.

34. Weber C, Podda M, Rallis M, Thiele JJ, Traber MG, Packer L. Efficacy of topically applied tocopherols and tocotrienols in protection of murine skin from oxidative damage induced by UV-irradiation. Free Radic Biol Med. 1997;22:761-69.

35. Musalmah M, Muhd Fairuz AH, Gapor MT, Wan Ngah WZ. Effect of palm vitamin $\mathrm{E}$ on wound healing in streptozotocin-induced diabetic rats. Malaysian J Biochem Mol Biol. 2001;6:34-9.

36. Ross R. The pathogenesis of atherosclerosis: a perspective for the $1990 \mathrm{~s}$ Nature. 1993;362:801-09.

37. Kamalakkannan N, Prince P. Hypoglycemic Effect Of Water Extracts Of Aegle Marmelos Fruits In Streptozotocin Diabetic Rats. J Ethnopharmacol. 2003;87:207-10.

38. Baynes JW, Thorpe SR. Role of oxidative stress in diabetic complications: a new perspective on an old paradigm. Diabetes. 1999;48:1-9. 
39. King GL, Loeken MR. Hyperglycemia-induced oxidative stress in diabetic complications. Histochem. Cell Biol. 2004;122:333-38.

40. Sasaki H, Nakamura J, Koh N, Hamada Y, Hara T, Chaya S, Nakashima E, Naruse K, Kato K, Kasuya Y, Sakakibara F, Hotta N. Effect of vitamin $\mathrm{E}$ and allylamine on the proliferation of cultured aortic smooth muscle cells from streptozotocin-induced diabetic rats. Life Sci. 1999;64:231725 .
41. Abdul Mutalib MS, Khaza'ai H, Wahle KW. Palm-tocotrienol rich fraction (TRF) is a more effective inhibitor of LDL oxidation and endothelial cell lipid peroxidation than alpha-tocopherol in vitro. Food Res Int. 2003;36:405-13.

42. Idris A, Sundram K. Palm vitamin E inhibits the progression of atherosclerosis in rabbit fed an atherogenic diet. Atherosclerosis. 2006;7:436. 